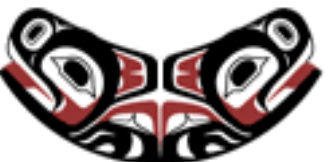

UW Biostatistics Working Paper Series

5-2-2007

\title{
Gamma Generalized Linear Models for Pharmacokinetic Data
}

\author{
Ruth Salway \\ University of Bath, UK, R.E.Salway@bath.ac.uk \\ Jon Wakefield \\ University of Washington, jonno@u.washington.edu
}

\section{Suggested Citation}

Salway, Ruth and Wakefield, Jon, "Gamma Generalized Linear Models for Pharmacokinetic Data" (May 2007). UW Biostatistics Working Paper Series. Working Paper 309.

http://biostats.bepress.com/uwbiostat/paper309

This working paper is hosted by The Berkeley Electronic Press (bepress) and may not be commercially reproduced without the permission of the copyright holder.

Copyright $(\odot) 2011$ by the authors 


\section{Introduction}

Pharmacokinetics is the study of the time course of a drug and its metabolites after introduction into the body. Pharmacokinetic studies are carried out extensively in drug development as they provide an invaluable aid in describing a drug's absorption, distribution and elimination characteristics.

In this paper we consider an alternative to the traditional compartment models used for pharmacokinetic data, based on a generalized linear model. Wakefield (2004) briefly considered such models for a single individual; here we provide more details and extend the approach to multiple individuals.

The structure of this paper is as follows. In Section 2 we review compartmental models and inference for such models. Sections 3 and 4 describe the log-linear gamma generalized linear models (GLMs) for single and multiple individuals, respectively. The use of these models is demonstrated in Section 5 and a concluding discussion is provided in Section 6.

\section{Compartmental Models}

Traditionally, to carry out statistical analysis of pharmacokinetic data the body is modeled as a system of compartments within which drug kinetics are assumed to be homogeneous, leading to concentration-time profiles that are sums of exponential. Gibaldi and Perrier (1982) provide a comprehensive account of pharmacokinetic models and principles, and Godfrey (1983) an account of compartmental modeling in general. Pharmacokinetic modeling via a compartmental system is a convenient visualization but the compartments often have no physiological meaning. Given the simplicity of such systems, the belief in the specific model chosen is often questioned, but certain physiological parameters are important for clinical application. For example the 
clearance, which is defined as the volume of blood that is cleared of drug in unit time, is of fundamental interest, as are the elimination half-life, that is the time taken for the drug concentration to drop by $50 \%$, and, for oral doses, the maximum concentration and the time to maximum concentration. The classic text due to Rowland and Tozer (1995) takes a "model-free" approach to pharmacokinetics in that the emphasis is on the physiological parameters, rather than on compartmental systems. Other authors (Weiss (1986), Weiss (1987)) have also strayed from compartmental models, and instead concentrated upon the estimation of model-free quantities of interest via a convenient representation.

For an intravenous bolus dose of size $D$, a $p$ compartment model with first-order kinetics leads to the time course of the concentration

$$
\mu(t)=\frac{D}{V} \sum_{k=1}^{p} A_{k} \exp \left(\beta_{k} t\right),
$$

a sum of exponentials. The parameter $V$ is an "apparent" volume that converts total amounts of drug to concentrations; it is an apparent volume since it may exceed the total volume of blood in the body due to binding, etc. The $A_{k}$ parameters describe the relative contributions of each of the $p$ exponentials. The stochastic part of the model includes measurement error and errors due to model misspecification. Let $y\left(t_{i j}\right)$ be the $j$ th measured concentration for the $i$ th individual at time $t_{i j}$. Assay precision is often found to increase with increasing true concentrations and models of the form

$$
y\left(t_{i j}\right)=\mu\left(t_{i j}\right)+\delta\left(t_{i j}\right)
$$

where $\delta\left(t_{i j}\right) \sim_{i n d} N\left\{0, \mu\left(t_{i j}\right)^{\gamma} \sigma_{\delta}^{2}\right\}$ with $\gamma>0$ have been used (e.g. Davidian and Giltinan (1995)). An alternative, with constant coefficient of variation, 


$$
\log y\left(t_{i j}\right)=\log \mu\left(t_{i j}\right)+\epsilon\left(t_{i j}\right)
$$

with $\epsilon\left(t_{i j}\right) \sim_{i i d} N\left(0, \sigma^{2}\right)$. Lindsey et al. (2000) have suggested combining a compartmental model mean with a gamma error model.

We consider in detail the one-compartment model with first-order absorption and elimination. Let $w_{k}(t)$ represent the amount of drug in compartment $k, k=0,1$ at time $t$, with compartment 0 representing the site from which absorption occurs; compartment 1 may be nominally thought of as corresponding to blood. The drug flow between the compartments is described by the differential equations

$$
\begin{aligned}
\frac{d w_{0}}{d t} & =-k_{a} w_{0}, \\
\frac{d w_{1}}{d t} & =k_{a} w_{0}-k_{e} w_{1}
\end{aligned}
$$

where $k_{a}$ is the absorption rate constant associated with the flow from compartment 0 to compartment 1 , and $k_{e}$ is the elimination rate constant. At time zero the initial dose is $w_{0}(0)=D$, and given $V$, the volume of distribution, we have

$$
\mu(t)=\frac{D k_{a}}{V\left(k_{a}-k_{e}\right)}\left\{\exp \left(-k_{e} t\right)-\exp \left(k_{a} t\right)\right\} .
$$

A common error model is:

$$
\log y_{i j}=\log \mu_{i j}+\epsilon_{i j}
$$

with $\epsilon_{i j} \sim_{i i d} N\left(0, \sigma^{2}\right)$, where $y_{i j}=y\left(t_{i j}\right)$ and $\mu_{i j}=\mu\left(t_{i j}\right)$. Model (2) is sometimes known as the "flip-flop" model because there is non-identifiability; the parameters $\left(V, k_{a}, k_{e}\right)$ give the same curve as the parameters $\left(V k_{e} / k_{a}, k_{e}, k_{a}\right)$. 
To enforce identifiability it is typical to assume that $k_{a}>k_{e}>0$. This identifiability issue become worse as the number of compartments increases.

The derived parameters of interest, given in terms of $\left(V, k_{a}, k_{e}\right)$, are:

- the time to maximum concentration:

$$
t_{\max }=\frac{1}{k_{a}-k_{e}} \log \left(\frac{k_{a}}{k_{e}}\right) ;
$$

- the maximum concentration:

$$
c_{\max }=\mu\left(t_{\max }\right)=\frac{D}{V}\left(\frac{k_{e}}{k_{a}}\right)^{k_{e} /\left(k_{a}-k_{e}\right)} ;
$$

- the clearance (initial dose divided by the area under the concentration curve):

$$
\mathrm{Cl}=V \times k_{e}
$$

- the elimination half-life:

$$
t_{1 / 2}=\frac{\log 2}{k_{e}}
$$

The first three quantities may be viewed as model-free in the sense that for any assumed functional concentration-time form they may be calculated. The half-life depends on the dominant term for large times being an exponential with positive $k_{e}$.

Model (2) is a nonlinear regression model and inference may proceed from either a frequentist or Bayesian perspective; Bates and Watts (1988) discuss in detail the intrinsic non-linearity of compartment models, which can lead to difficulties in both computation and asymptotic inference. For a Bayesian approach (which may be essential if only sparse data is available on a particular individual, Wakefield (1996)) we advocate prior specification 
in terms of the derived parameters of interest. Such priors are easier to specify, though one disadvantage is that the transformation from priors on derived parameters to model parameters may require numerical root-solving which complicates computation. For example, this problem arises for the transformation $\left(c_{\max }, t_{\max }, t_{1 / 2}\right) \rightarrow\left(V, k_{a}, k_{e}\right)$.

\section{Gamma Generalized Linear Models for Single Individuals}

\subsection{Model Description}

We can rewrite the one-compartment model (2) as

$$
\mu(t)=D \exp \left(\beta_{0}+\beta_{1} t\right)\left[1-\exp \left\{-\left(k_{a}-k_{e}\right) t\right\}\right]
$$

where $\beta_{0}=\log \left\{k_{a} /\left[V\left(k_{a}-k_{e}\right)\right]\right\}$ and $\beta_{1}=-k_{e}$. This suggests an alternative to the compartment model, using a GLM and fitting the log-linear fractional polynomial model:

$$
E\left(y_{i j}\right)=\mu_{i j}=D_{i} \exp \left(\beta_{0}+\beta_{1} t_{i j}+\beta_{2} / t_{i j}\right)
$$

Here, $\beta_{2}$ determines the absorption, and we require $\beta_{1}<0$ and $\beta_{2}<0$ to ensure an increasing absorption phase and a decreasing elimination phase. The data are assumed to be gamma distributed, with $y_{i j} \sim \operatorname{Ga}\left\{\phi^{-1},\left(\mu_{i j} \phi\right)^{-1}\right\}$ so that $\phi^{1 / 2}$ is the coefficient of variation.

Again, we are interested in the derived parameters, expressed in terms of $\left(\beta_{0}, \beta_{1}, \beta_{2}\right)$ :

- the time to maximum concentration:

$$
t_{\max }=\left(\frac{\beta_{2}}{\beta_{1}}\right)^{1 / 2} ;
$$

- the maximum concentration:

$$
c_{\max }=D \exp \left[\beta_{0}-2\left\{\beta_{1} \beta_{2}\right)^{1 / 2}\right\}
$$


- the clearance:

$$
\mathrm{Cl}=\frac{\left(\beta_{1} / \beta_{2}\right)^{1 / 2}}{2 \exp \left(\beta_{0}\right) K_{1}\left[2\left(\beta_{1} \beta_{2}\right)^{1 / 2}\right]} ;
$$

where $K_{n}[x]$ denotes a modified Bessel function of the second kind of order $n$.

- the elimination half-life:

$$
t_{1 / 2}=-\frac{\log 2}{\beta_{1}} .
$$

\subsection{Likelihood Inference}

Since the form of (3) is a generalized linear model, estimation is straightforward using maximum likelihood for the fixed parameters, and a moment estimator for the dispersion parameter.

Confidence intervals for the derived parameters of interest may be based on the asymptotic distribution of the MLE using the delta method on the $\log$ of the parameters; the variances of these are provided in the appendix.

\subsection{Bayesian Inference}

We would rather place priors on the derived parameters of interest since pharmacokineticists find these more straightforward to think about and may combine information from a variety of sources, and not just on previous compartmental analyses. Model comparisons of mean models and error distributions via Bayes factors are also more interpretable since the same priors may be used across models.

The posterior distribution is not tractable and so we resort to simulationbased methods. A convenient way to implement the Bayesian approach for low-dimensional posteriors with informative priors is via a rejection algorithm which takes as generating density the prior distribution (Wakefield 
(1994)). This approach produces independent samples from the posterior and so can be automated since it does not require subjective assessment of burn-in or convergence. Unlike Markov chain Monte Carlo (MCMC) it also allows straightforward calculation of Bayes factors for model comparison.

Let $\boldsymbol{\theta}$ denoted the parameters, for example, the collection $\left(t_{1 / 2}, t_{\max }, c_{\max }\right.$, $\mathrm{cv})$, with prior distribution $\pi(\boldsymbol{\theta})$, and let $M=\sup _{\boldsymbol{\theta}} p(\mathbf{y} \mid \boldsymbol{\theta})$ be the maximized likelihood. Then the rejection algorithm proceeds as follows:

1. Generate $U \sim U(0,1)$ and $\boldsymbol{\theta} \sim \pi(\boldsymbol{\theta})$, independently.

2. Accept $\boldsymbol{\theta}$ if

$$
U<\frac{p(\mathbf{y} \mid \boldsymbol{\theta})}{M}
$$

otherwise reject $\boldsymbol{\theta}$.

\section{Return to 1.}

This approach requires the prior to be proper; proper priors are in general needed for compartmental models in order to ensure a proper posterior distribution. The acceptance probability of the algorithm is $p_{a}=p(\mathbf{y}) / M$, so an estimate of the prior predictive is

$$
\widetilde{p}(\mathbf{y})=M \widehat{p}_{a}
$$

To compare models $M_{0}$ and $M_{1}$, the Bayes factor is

$$
\frac{p\left(\mathbf{y} \mid M_{0}\right)}{p\left(\mathbf{y} \mid M_{1}\right)}
$$

the ratio of the marginal distributions of the data under the two models. An estimate of the marginal distribution can be obtained for each model using 
the observed acceptance rate via (5). Alternatively, an importance sampling algorithm can be used; see Pauler, Wakefield and Kass (1999) for details.

Implementation via the rejection algorithm is straightforward for the GLM, but for the compartment model requires root-solving to find $k_{a}$, increasing the computational burden. The samples may be transformed for other quantities of interest, for example, the clearance can be calculated directly via substitution of $\boldsymbol{\beta}$ samples into (4)

\section{Gamma Generalized Linear Models for Multiple Individuals}

\subsection{Model Description}

For multiple individuals we may use a generalized linear mixed model (GLMM):

$$
\begin{aligned}
y_{i j} & \sim \operatorname{Ga}\left\{\phi^{-1},\left(\mu_{i j} \phi\right)^{-1}\right\} \\
\mu_{i j} & =D_{i} \exp \left(\beta_{i 0}+\beta_{i 1} t_{i j}+\beta_{i 2} / t_{i j}\right) \\
\boldsymbol{\beta}_{i} & \sim N(\boldsymbol{\beta}, \boldsymbol{\Sigma})
\end{aligned}
$$

where $\boldsymbol{\beta}_{i}=\left(\beta_{i 0}, \beta_{i 1}, \beta_{i 2}\right)^{T}$ and $\boldsymbol{\Sigma}$ is a $3 \times 3$ covariance matrix.

Here we have specified random effects for each element of $\boldsymbol{\beta}$. However, we require $\beta_{i 0}>0, \beta_{i 1}<0, \beta_{i 2}<0$ for the fitted curve to be meaningful; one possibility is to re-parameterize and specify normal random effects for $\left\{\beta_{i 0}, \log \left(-\beta_{i 1}\right), \log \left(-\beta_{i 2}\right)\right\}$.

\subsection{Likelihood Inference}

Model (8) is a straightforward GLMM and so can fitted using standard software, for example, the lmer() function in R. Unfortunately, if we need to re-parameterize the model it is no longer a GLM, and we need to use a more general random effects fitting procedure (such as nlme() in R). Thus 
we lose one of the benefits of this formulation over the compartment model.

\subsection{Bayesian Inference}

In the Bayesian framework the illegal values in the parameter space can be avoided using the prior, though the normality assumption of random effects may be more reasonable on a transformed scale. It is perhaps more natural to specify the random effects on the parameters of interest and so we combine (6) and (7) with the alternative random effects model:

$$
\log \boldsymbol{\theta}_{i} \sim N(\boldsymbol{\mu}, \Sigma)
$$

where $\boldsymbol{\theta}_{i}=f\left(\boldsymbol{\beta}_{i}\right)=\left(t_{i 1 / 2}, t_{i \max }, c_{i \max }\right)^{T}$ transforms the likelihood parameterization $\boldsymbol{\beta}_{i}$ to the prior parameterization $\boldsymbol{\theta}_{i}$. The model is completed with priors for $\phi, \boldsymbol{\mu}$ and $\boldsymbol{\Sigma}$.

The rejection algorithm described for the fixed effects model is no longer feasible for this model, as the acceptance probabilities are too small. However, samples may be generated using an MCMC algorithm. For example, it is straightforward to express this model in WinBUGS (Spiegalhalter et al. (2003)).

\section{Application}

We illustrate the models using data on 12 subjects given an oral dose of the anti-asthmatic agent theophylline, with 10 concentration measurements obtained from each individual over the subsequent 25 hours. The data are shown in Figure 1; they were originally analyzed in Upton et al. (1982) and are available from the Resource Facility for Population Kinetics at http://www.rfpk. washington. edu.

[Figure 1 about here.] 


\subsection{Individual Fitting}

To compare the compartment and GLM models, we fit the single models to each of the twelve individuals. The fitted curves in Figure 1, corresponding to the MLEs for the compartmental and generalized linear models, are very similar. The computation time for the frequentist models was slightly greater for the GLM, though the fitting for all 12 individuals was negligible (less than a third of a second for both model classes). For the Bayesian models we used independent log-normal priors for $t_{1 / 2}, t_{\max }, c_{\max }$, cv, with medians $8,1.5$, 9, 0.1 respectively and $90^{\text {th }}$ percentiles $26,6,36,10$. We calculated Bayes factors, as a side-product of the rejection method; using the guidelines given in Kass and Raftery (1995) six individuals have positive evidence in favor of the GLM, and two have positive evidence in favor of the compartmental model, so that neither model is consistently preferred. Marginal inference for the derived parameters of interest was consistent across models; for example, Figure 2 shows similar histogram posterior representations for $t_{1 / 2}, t_{\max }$ and clearance for the first individual.

[Figure 2 about here.]

Plots of the likelihood surfaces for the frequentist models (Figure 3) show more regular elliptical surfaces for the GLM model which suggests that asymptotic inference would be more reliable for the GLM. Although we should be wary of generalizing these results, there seems to be little difference between the two models in terms of fit, with some possible advantages to the GLM model.

[Figure 3 about here.] 
In fitting the frequentist models, one individual produced invalid parameter estimates under the GLM, with $\beta_{2}$ slightly greater than zero. On closer examination we found that this individual had a different profile from the others, with a much higher initial concentration, and lower time to maximum concentration, suggesting the model may be inappropriate for this individual. In the Bayesian analysis the problem of positive $\beta_{2}$ was avoided because the prior excluded such possibilities.

\subsection{Population Fitting}

We fitted generalized linear and non-linear mixed effects models, with all parameters random; results are summarized in Table 1. For the Bayesian models we used independent lognormal priors on the fixed effects, with median and $90^{\text {th }}$ percentile as in the individual fitting, and an inverse Wishart

prior for $\boldsymbol{\Sigma}^{-1}$, which requires specification of a scale matrix and a degrees of freedom. For the former we choose a diagonal matrix with elements 0.04, which corresponds to $20 \%$ coefficient of variation for the parameters on the non-logarithmic scale (Wakefield et al. (1994)). Lower degrees of freedom correspond to flatter distributions and so we chose a value of three, which is the lowest value that produces a proper posterior. The GLMM ran much faster than the non-linear MEM; 0.66 seconds versus 36.5 seconds. The Bayesian models are more difficult to compare since the GLMM was implemented in WinBUGS while the compartmental model required specific $\mathrm{R}$ code to be written (because of the need for root-solving).

[Table 1 about here.]

The frequentist GLMM gives slightly different results to the other models, since the random effects are normal on the $\boldsymbol{\beta}$ scale, rather than log-normal. 
As before, there is little difference between estimates from the GLM and compartment models. With only 12 individuals we might question the sensitivity to the scale matrix in the Wishart distribution. For this example, however, inference was relatively insensitive to this choice. When we chose diagonal elements 0.01 and 0.25 (corresponding to approximate coefficients of variation of $10 \%$ and $50 \%$, respectively); the medians were largely unchanged and the interval estimates moved in the expected directions. For example, for the GLMM the biggest changes were for $t_{1 / 2}$ and $c_{\max }$. For $t_{1 / 2}$ the interval estimates were $(7.04,8.33),(6.99,8.42),(6.83,8.71)$ under diagonal elements $0.01,0.04,0.25$, and for $c_{\max }$ the corresponding estimates were $(8.08,9.64)$, $(8.05,9.70),(7.83,9.92)$.

\section{Discussion}

We have presented an alternative formulation to compartment models for pharmacokinetic data with single doses. If multiple doses are considered the mean function does not correspond to a GLM and so the models lose a lot of their appeal. We would also not advocate the use of the GLM mean function for predicting pharmacokinetic behavior under multiple doses, since the steady-state behavior has not been investigated.

An advantage of the GLM is that it is generally easier to fit. For single individuals it takes the form of a standard GLM, and likelihood surfaces are more regular. From a computational point of view the Bayesian models benefit from the straightforward conversion between the $\boldsymbol{\beta}$ model parameters and $\boldsymbol{\theta}$ the derived parameters of interest; the compartment model needs to use numerical root-solving for this conversion.

A disadvantage of the GLM model is that it is only interpretable for 
$\beta_{1}<0, \beta_{2}<0$, and it is possible to obtain estimates that do not correspond to real-valued estimates of the parameters of interest. When we observed this in our data it seemed to indicate that the model was inappropriate, suggesting model refinement was required. The problem of uninterpretable estimates may be overcome in the Bayesian approach by placing priors directly on the parameters of interest, since this imposes the constraints as illegal values have no support under the prior. We can reparameterize for the frequentist approach, but the model is no longer a GLM though retains the advantage of being able to easily convert between parameterizations without requiring root-solving.

This paper has concentrated on the GLM as an alternative to the onecompartment model with first-order absorption. However, the approach may easily be extended to mimic the behavior of multiple compartment models by inclusion of extra terms, for example $t^{-2}$ and/or $t^{-1 / 2}$. We might for example consider a model of the form

$$
\mu(t)=D \exp \left(\beta_{0}+\beta_{1} t+\beta_{2} / t+\beta_{3} / t^{2}\right)
$$

The aim is to provide a simple class of model that mimic the qualitative behavior of compartmental models. For any given GLM formulation we can calculate the parameters of interest, though this may require numerical methods. We should take care to ensure that the area under the curve is finite for any prospective model, to ensure finite clearance. For example, the canonical reciprocal link does not provide a finite area when combined with mean function (3).

For compartment models it is common to model volume of distribution as a function of covariates, such as weight. The term $\exp \left(\beta_{0}\right)$ is related to 
the volume, suggesting that covariates could be included in $\beta_{0}$ for the GLM formulation. Thus an obvious extension is to incorporate covariates $Z$ in the natural way, by multiplying the mean concentration by $\exp \left(\gamma^{T} Z\right)$.

\section{Appendix}

We give the variances for the logs of estimators of parameters of interest in the one-compartment model:

$$
\begin{aligned}
\operatorname{var}(\log \widehat{c v}) \approx & \frac{\phi^{2} \operatorname{var}\left(\phi^{-1}\right)}{4} \\
\operatorname{var}\left(\log \widehat{t}_{1 / 2}\right) \approx & \frac{\operatorname{var}\left(\beta_{1}\right)}{\beta_{1}^{2}} \\
\operatorname{var}\left(\log \widehat{t}_{\text {max }}\right) \approx & \frac{\operatorname{var}\left(\beta_{1}\right)}{4 \beta_{1}^{2}}+\frac{\operatorname{var}\left(\beta_{2}\right)}{4 \beta_{2}^{2}}-\frac{\operatorname{cov}\left(\beta_{1}, \beta_{2}\right)}{4 \beta_{1} \beta_{2}} \\
\operatorname{var}\left(\log \widehat{c}_{\text {max }}\right) \approx & \operatorname{var}\left(\beta_{0}\right)+\left(\frac{\beta_{2}}{\beta_{1}}\right) \operatorname{var}\left(\beta_{1}\right)+\left(\frac{\beta_{1}}{\beta_{2}}\right) \operatorname{var}\left(\beta_{2}\right)+2 \operatorname{cov}\left(\beta_{1}, \beta_{2}\right) \\
& -2\left(\frac{\beta_{2}}{\beta_{1}}\right)^{1 / 2} \operatorname{cov}\left(\beta_{0}, \beta_{1}\right)-2\left(\frac{\beta_{1}}{\beta_{2}}\right)^{1 / 2} \operatorname{cov}\left(\beta_{0}, \beta_{2}\right)
\end{aligned}
$$

The variance for clearance is more complex but can be calculated as

$$
\begin{aligned}
D_{1}= & \frac{1}{\beta_{1}}-\left(\frac{\beta_{2}}{\beta_{1}}\right)^{1 / 2} \frac{K_{0}\left[2\left(\beta_{1} \beta_{2}\right)^{1 / 2}\right]}{K_{1}\left[2\left(\beta_{1} \beta_{2}\right)^{1 / 2}\right]} \\
D_{2}= & -\left(\frac{\beta_{1}}{\beta_{2}}\right)^{1 / 2} \frac{K_{0}\left[2\left(\beta_{1} \beta_{2}\right)^{1 / 2}\right]}{K_{1}\left[2\left(\beta_{1} \beta_{2}\right)^{1 / 2}\right]} \\
\operatorname{var}(\log \widehat{\mathrm{Cl}})= & \operatorname{var}\left(\beta_{0}\right)+D_{1}^{2} \operatorname{var}\left(\beta_{1}\right)+D_{2}^{2} \operatorname{var}\left(\beta_{2}\right)-2 D_{1} \operatorname{cov}\left(\beta_{0}, \beta_{1}\right) \\
& -2 D_{2} \operatorname{cov}\left(\beta_{0} \beta_{2}\right)+2 D_{1} D_{2} \operatorname{cov}\left(\beta_{1}, \beta_{2}\right)
\end{aligned}
$$

\section{ACKNOWLEDGEMENTS}

The work of the first author is supported by the Bath Institute for Complex Systems (EPSRC Grant GR/S86525/01). The dataset used in this paper is made available by the National Institutes of Health grant EB-01975. The 
authors appreciate the constructive comments of an associate editor and two referees.

\section{REFERENCES}

Bates, D. and Watts, D. (1988). Non-linear Regression Analysis and Its Applications. Wiley.

Davidian, M. and Giltinan, D. (1995). Nonlinear Models for Repeated Measurement Data. Chapman and Hall, London.

Gibaldi, M. and Perrier, D. (1982). Drugs and the Pharmaceutical Sciences, Volume 15: Pharmacokinetics, Second Edition. Marcel Dekker.

Godfrey, K. (1983). Compartmental Models and their Applications. Academic Press, London.

Kass, R. and Raftery, A. E. (1995). Bayes factors. Journal of the American Statistical Association 90, 773-95.

Lindsey, J., Byrom, W., Wang, J., Jarvis, P. and Jones, B. (2000). Generalized nonlinear models for pharmacokinetic data. Biometrics 56, 81-88.

Pauler, D., Wakefield, J. and Kass, R. (1999). Bayes factors for variance component models. Journal of the American Statistical Association 94, $1242-53$.

Rowland, M. and Tozer, T. (1995). Clinical Pharmacokinetics, Third Edition. Williams and Wilkins.

Spiegalhalter, D., Thomas, A., Best, N. and Lunn, D. (2003). WinBUGS User Manual Version 1.4. Medical Research Council Biostatistics Unit, Cambridge.

Upton, R., Thiercelin, J. F., Guentert, T. W., Wallace, S., Powell, J., San- 
som, L. and Riegelman, S. (1982). Intraindividual variability in theophylline pharmacokinetics: statistical verification in 39 of 60 healthy young adults. Journal of Pharmacokinetics and Biopharmaceutics 10, $123-34$.

Wakefield, J. (1994). An expected loss approach to the design of dosage regimens via sampling-based methods. The Statistician 43, 13-29.

Wakefield, J. (1996). Bayesian individualization via sampling-based methods. Journal of Pharmacokinetics and Biopharmaceutics 24, 103-131.

Wakefield, J. (2004). Non-linear regression modelling and inference. In Adams, N., Crowder, M., Hand, D. and Stephens, D., editors, Methods and Models in Statistics, pages 119-153. Imperial College Press, London.

Wakefield, J., Smith, A., Racine-Poon, A. and Gelfand, A. (1994). Bayesian analysis of linear and non-linear population models using the gibbs sampler. Applied Statistics 43, 201-221.

Weiss, M. (1986). Generalizations in linear pharmacokinetics using properties of certain classes of residence time distributions. I. Log-convex drug disposition curves. Journal of Pharmacokinertics and Biopharmaceutics 14, 635-657.

Weiss, M. (1987). Generalizations in linear pharmacokinetics using properties of certain classes of residence time distributions. II. Log-concave concentration-time curves following oral administration. Journal of Pharmacokinertics and Biopharmaceutics 15, 57-74. 

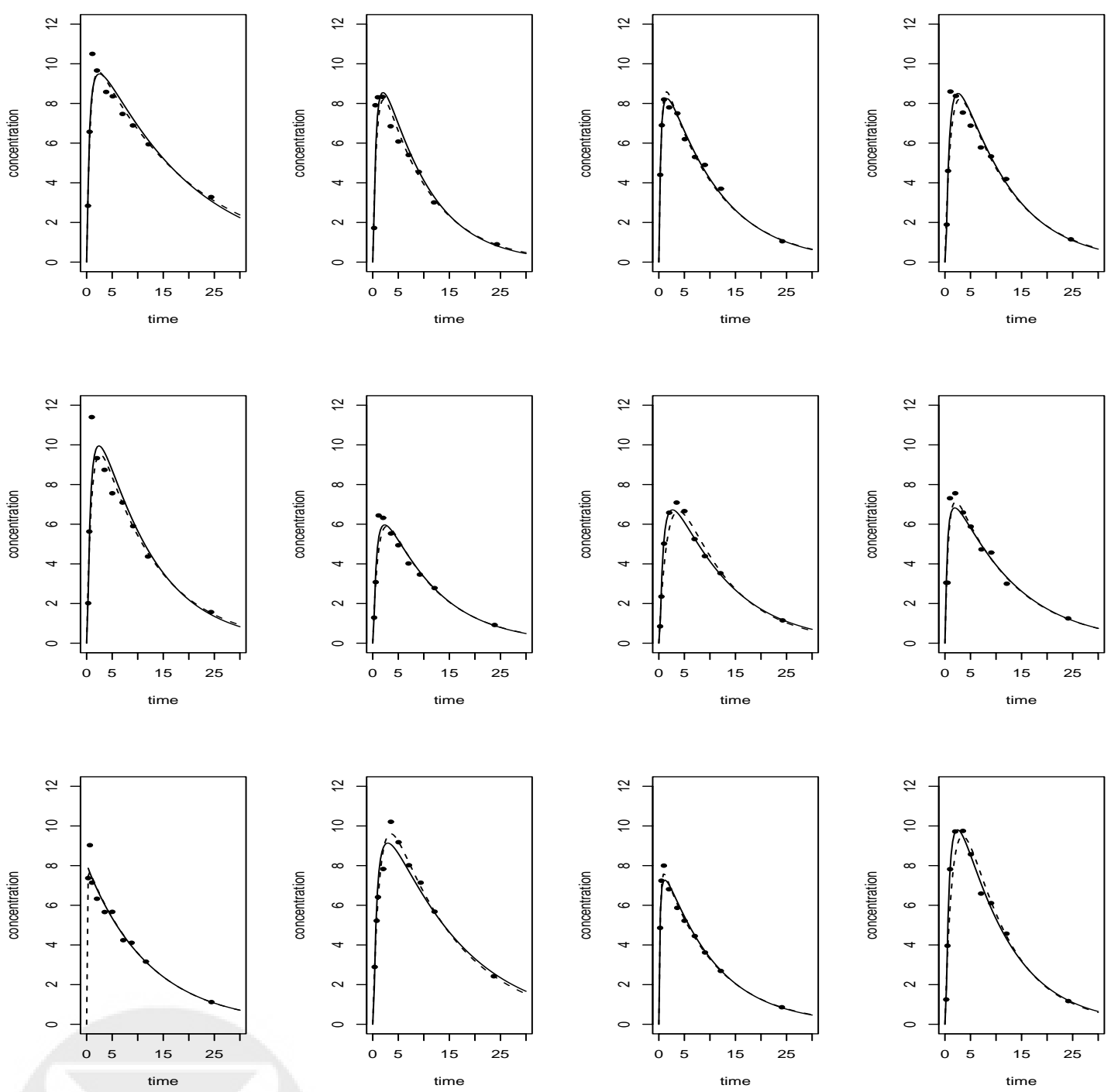

Figure 1. Concentrations of Theophylline versus time for 12 individuals, along with fitted curves; the solid lines correspond to the GLM and the dashed lines to the compartmental model. 
07

$\cdot($ мол шот7оq)

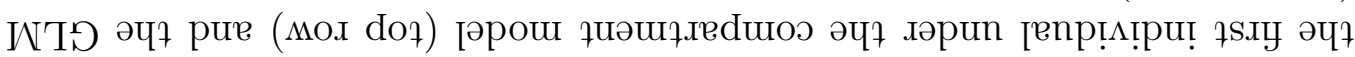

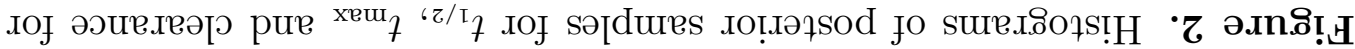
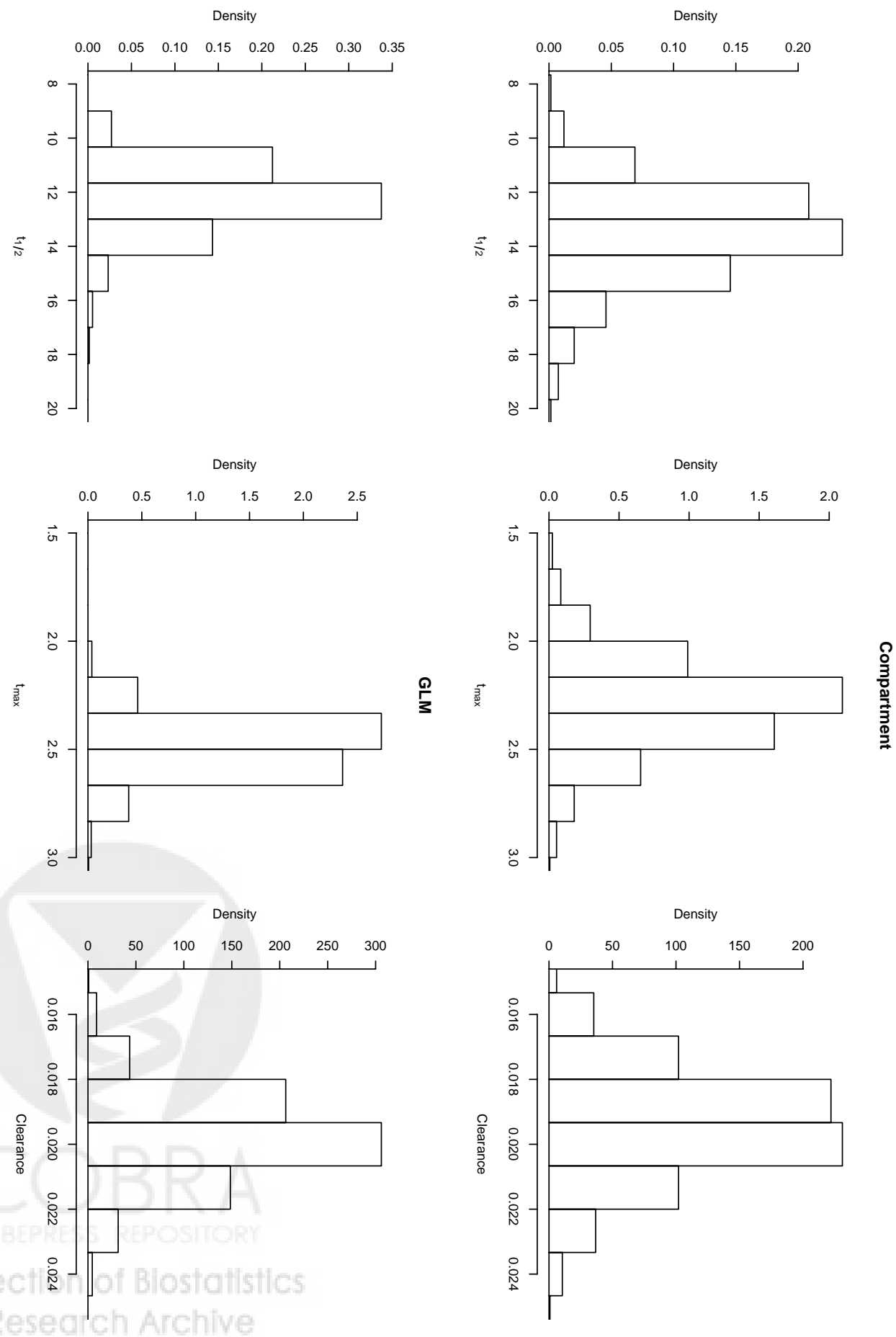

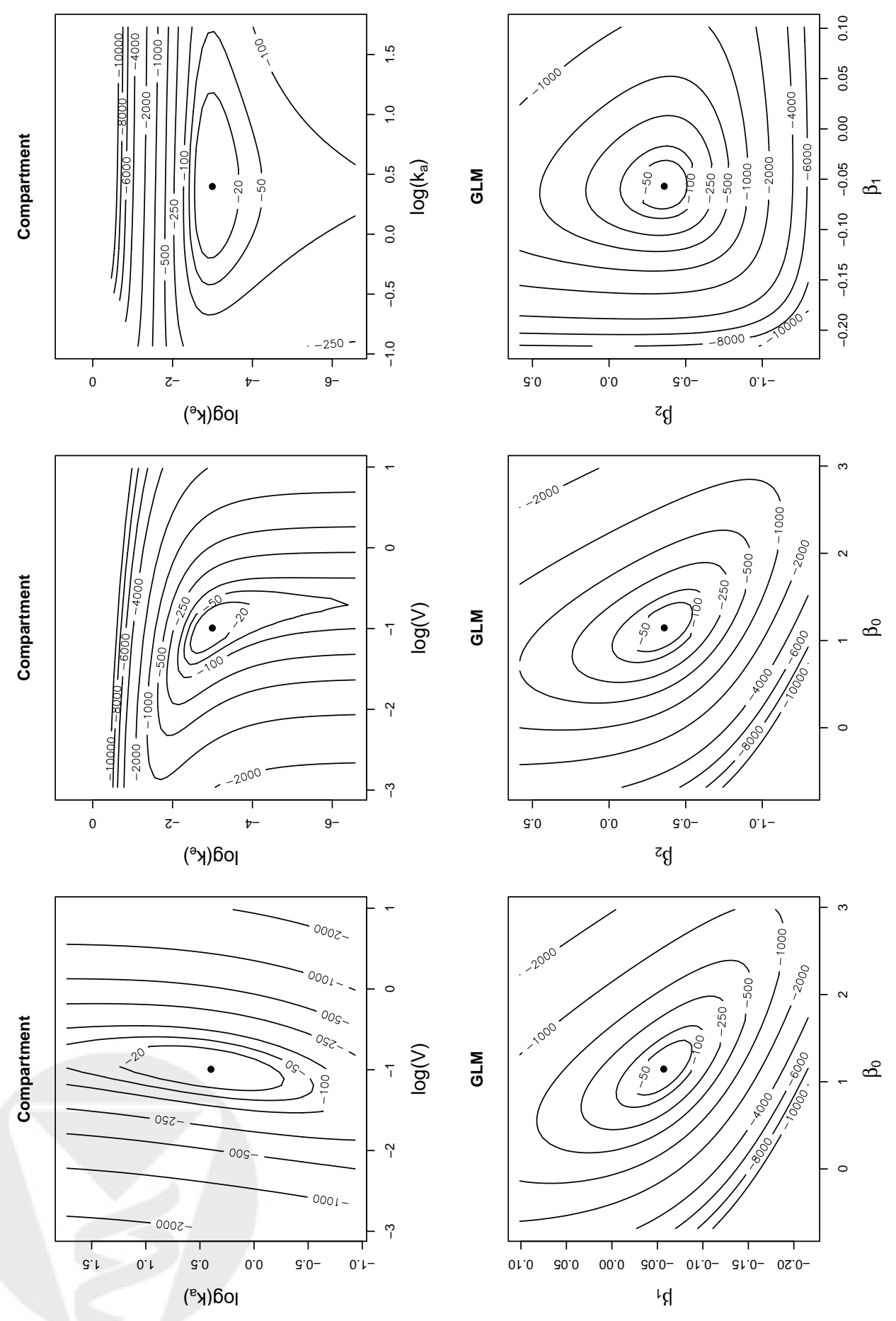

Figure 3. Likelihood surfaces for the first individual for the compartment model and the GLM. 
Table 1

Comparison of fixed effects for mixed effects analyses: parameter estimates (for the Bayesian analyses posterior medians are given) and $95 \%$ interval estimates. Estimates for the maximum concentration are for a dose of $5 \mathrm{mg}$.

\begin{tabular}{|c|c|c|c|c|}
\hline \multirow[b]{2}{*}{ Parameter } & \multicolumn{2}{|c|}{ Compartment } & \multicolumn{2}{|r|}{ GLM } \\
\hline & Fixed effect & (95\% interval) & Fixed effect & (95\% interval) \\
\hline \multicolumn{5}{|c|}{ FREQUENTIST } \\
\hline$t_{1 / 2}$ & 7.75 & $(7.06,8.51)$ & 7.58 & $(6.88,8.35)$ \\
\hline$t_{\max }$ & 2.26 & $(1.73,2.96)$ & 2.17 & $(1.89,2.49)$ \\
\hline$c_{\max }$ & 9.06 & $(8.15,10.1)$ & 8.67 & $(7.74,9.71)$ \\
\hline $\mathrm{Cl} \times 10^{3}$ & 40.3 & $(35.0,46.4)$ & 40.5 & $(35.0,46.9)$ \\
\hline $\mathrm{cv} \times 10^{2}$ & 17.1 & $(15.1,19.5)$ & 10.8 & $(9.5,12.2)$ \\
\hline \multicolumn{5}{|c|}{ BAYESIAN } \\
\hline$t_{1 / 2}$ & 7.78 & $(6.80,8.72)$ & 7.66 & $(6.99,8.42)$ \\
\hline$t_{\max }$ & 2.21 & $(1.65,2.85)$ & 2.01 & $(1.68,2.38)$ \\
\hline$c_{\max }$ & 8.73 & $(7.72,10.0)$ & 8.82 & $(8.05,9.70)$ \\
\hline $\mathrm{Cl} \times 10^{3}$ & 41.9 & $(32.4,54.6)$ & 39.8 & $(38.7,40.8)$ \\
\hline $\mathrm{cv} \times 10^{2}$ & 17.2 & $(15.3,19.8)$ & 12.9 & $(11.4,14.8)$ \\
\hline
\end{tabular}

